\title{
Oral Hygiene Practices and Knowledge about Dental Caries among 15 years Old School Students in Lahore Population : A Cross Sectional Survey
}

\author{
Rasul $\mathrm{F}^{2 *}$, Rizvi Z $\mathrm{Z}^{2}$, Aqeel $\mathrm{R}^{3}$, Wahid $\mathrm{SM}^{2}$, Ahmed $\mathrm{A}^{\mathbf{1}}$ and Shah $\mathrm{SH}^{4}$ \\ ${ }^{1}$ Department of Oral Biology de' Montmorency College of Dentistry, Pakistan \\ ${ }^{2}$ Department of Oral Pathology de' Montmorency College of Dentistry, Pakistan \\ ${ }^{3}$ Department of Oral Pathology Lahore Medical Dental College, Lahore \\ ${ }^{4}$ Department of Orthodontics de' Montmorency College of Dentistry, Pakistan \\ *Corresponding author: Faiz Rasul, Demonstrator/Lecturer Oral Pathology, de' Montmorency College of Dentistry, Lahore, \\ Pakistan
}

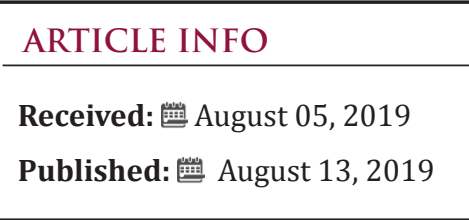

Citation: Rasul F, Rizvi Z, Aqeel R, Wahid SM, Ahmed A, Shah SH. Oral Hygiene Practices and Knowledge about Dental Caries among 15 years Old School Students in Lahore Population : A Cross Sectional Survey. Biomed J Sci \& Tech Res 20(3)-2019. BJSTR. MS.ID.003469.

Keywords: Dental Caries; Oral Hygiene Practices; Knowledge
ABSTRACT

Background: Dental Caries is one of the most prevalent chronic diseases in childhood in developed and underdeveloped countries. As oral hygiene, dietary habits, behavioral factors, sociodemographic factors, awareness of the disease and its prevention are concerned, all are important to be known by an individual of a community or society. Fifteen years age group adolescents have erupted all permanent teeth in the oral cavity and this age group children are developing their habits, so these habits will persist through out their life. It was important to know their oral hygiene habits and the concept about the dental caries. It is beneficial to identify children at increased risk and associated predictive factors of caries and tooth brushing in childhood and adolescence in our population. This study was designed to determine the prevalence of dental caries (DMFT), oral hygiene practices and knowledge about tooth decay in 15 years age school going children.

Methods: After the approval of institutional review board this study was conducted in the schools of Lahore having summer camp in July 2015. Study participants were school children, aged 15 years. Students of 9 th and 10 th classes attending summer camp were the sample of the study. A total 60 respondents were included in the study. Written permission was taken before their examination and interview. A self-structured questionnaire was used to collect information regarding oral hygiene practices and knowledge about the dental caries. Data entered and analyzed using SPSS version 20. Data was assessed by percentage and frequency, descriptive part was used for demographic variables, oral hygiene practices. To records the caries experience, Decayed, Missing, filled (DMF) caries index was used. The impact of all variables on status of caries was also analyzed by cross tabulation. To see impact of demographic variable, parents' education was selected as important variable. Chi-Square test of association was performed to see the relationship between father education and caries status. This regression was performed by using backward elimination method at five steps as Logistic Regression for the Prediction of Caries Status on Basis of oral hygiene Habits. P-value $\leq 0.05$ was taken as significant.

Results: Total participants were sixty as $n=60$.Majority (80\%) of the subjects or participants of the study were males and remaining were females. Total fifty-eight-point three percent $(58.3 \%)$ respondents were caries free in this study while caries positive were $41.7 \%$. Total decayed teeth were $61 \%$, Missed teeth were 04 and total Filled (restored) teeth were $07 \%$ in the sample. Average DMFT score examined was 1.2. Among the respondents $43.3 \%$ said that they used to brush once a day, $48.3 \%$ twice a day and only 1 person had not the habit of doing brush. Similarly, only $5 \%$ had the habit of dental floss once or more times a day. Only 23.3\% respondents were involved in doing Miswak once or twice a day. Very few number $11.7 \%$ were involved in using Manjan once or twice a day. But on the other side, $58.3 \%$ had the habit of water rinsing three or more times a day. Maximum number of persons involved in cleaning with tongue musculature was $15 \%$ 
which were doing that thrice a day. $80 \%$ subjects said that they knew that what is tooth decay and $96.7 \%$ replied that they had idea about its few common causes. For Teeth decay management people prefer to go to private hospital as recorded in this study, because $2 / 3$ rd of sample used to go to private hospitals. Almost every subject (92\%) answered that they knew what the negative impact this this caries created negative impact on their life and its quality. Chi square test of association has shown that higher the level of education, lower will be the chances of decay and vice versa. Similarly, mother education has shown same impact on the caries status. This relationship was also significant at prescribed level of significance. Lower financial status was linked with higher incidence of caries. Total 16 students out of 25 having caries belong to lower financial status. Three significant variables i.e., brushing after every meal, usage of Miswak and brush after dinner were playing significant protective role from teeth decay. Final logistic model will be $\mathrm{Z}=10.503$ 2.602 (Brush after every meal)-2.391 (usage of Miswak)- 2.320 (Brush after Dinner).

Conclusion: Findings of this study have shown that brushing twice a day and after every meal, brushing after dinner and before sleeping, using Miswak and having awareness about teeth protection or brushing were the significant data or practices for avoiding teeth decay. This study has explored that father's and mother's education level has strong positive impact on caries. Similarly, higher the financial position, lower are the incidence of dental caries.

\section{Introduction}

Dental caries is an important public health problem across the world [1]. It affects school children $60-90 \%$ reported by the World Health Organization (WHO) [2]. Burden of dental caries is declined in developed countries due to public health interventions such as water fluoridation, healthier lifestyles, and improved oral hygiene practices, however its burden is still high in developing countries and in Asia [1]. Oral Health Education intervention is effective in enhancing knowledge, attitude, and practices toward positive oral health [3] .Consequences of dental caries can be major public health issue such as tooth loss and impaired quality of life. This burden is due to structure of society, living conditions, lifestyles, and the existence of preventive oral health systems [4]. Different strata of population have different caries prevalence and prevalence of dental caries is more observed in the underprivileged population groups, such as people with low literacy rate, poor living conditions, and poor oral hygiene practices and with poor health seeking behavior $[1,5]$ Reduction in prevalence of dental caries is observed by introducing effective brushing in school children [6].

Good oral hygiene is an important primary preventive procedure that can prevent dental caries. Children, who brush their teeth less than once a day, and do not use fluoride toothpastes, have a higher risk of dental caries [7] A suitable oral health behavior during childhood perpetuates more effectively into adulthood. School going children have little knowledge about oral health in developing countries [8]. Plaque removal is an important element of oral health in order to prevent dental caries,[9] by toothbrushes, rinses, floss, and dentifrices containing fluoride $[10,11]$. Oral health status depends on a dynamic interplay of several factors, including the individual's personal attribute, behavior, and perception. It is of relevant concern for oral health professionals to impart a positive oral health knowledge and behavior in the society in order to promote health and reduce burden of disease [12]. Modifiable risk behaviors such as oral hygiene practices, dietary habits, and basic knowledge about the disease are considered important. An appropriate range of policies, strategies and approaches to disease prevention and health promotion can be generated only if a range of risks is known [13]. The aim of this study was to measure prevalence of dental caries in fifteen years old school students and to assess their oral hygiene practices. Furthermore, their knowledge regarding dental caries was also assessed.

\section{Material and Methods}

A cross sectional survey was conducted in summer camp of schools in Lahore in July 2015 after the approval of institutional review board. Lahore is the provincial capital of Punjab Province. Study participants were school children, aged 15 year of 9th and 10th classes. Written and verbal consent was taken from the students. Those who gave their consent were taken into the study. Study participants were examined by two dentists using for dental caries using DMFT index. Dental Caries were recorded as present when a carious cavity was apparent on visual inspection under examination light, mouth mirror and dental probe. If doubt existed in arrested caries and active lesion, the surface was investigated with a probe. Unless the point entered the cavitated lesion, the surface was recorded as sound. The catching of the probe in a pit and fissure was not enough to warrant the diagnosis of caries unless there was additional visual evidence. Average DMFT was calculated. Demographic characteristics were recorded such as identification number, age, gender, parents' education level, financial status or income of family, house ownership and availability of flowing water were asked from the respondent.

Parents' education was asked as Low (less than 10 years of education), Medium (11-12 years of education) and High (more 
than 12 years of education). Family income was also asked in Pakistani currency as less than Rs. 15000, between Rs.15001-50000 and more Rs. > 50001. Finally, availability of water was also asked. All of these demographics' variables were noted on individual data Performa. Oral hygiene habits mean practices of an individual for oral cleanliness. These practices include tooth brushing with paste, Miswak, powder (Manjan), dental floss, tongue musculature, rinses with water and combination of any two methods. About these practices two options were given to respondents as yes or no. Each practice was asked one by one from the respondents. Timings of teeth cleanings were also asked from the respondents in seven categories like teeth cleaning before breakfast, after breakfast, before lunch, after lunch, before dinner or after dinner and before nimaz. Last component was frequency of teeth cleanings like once a day, twice a day, three times or more and none.

All tools of oral hygiene practices like tooth brushing with paste, Miswak, powder and Manjan, dental floss, tongue musculature was asked in four options such as once a day, twice a day, three time or more and none. Similarly, thirty-four different questions were asked from respondent about the oral hygiene practices who gave answer as yes or no. Final part of the questionnaire was knowledge about the dental caries. Fifteen questions about this theme were asked from the respondents. These answers were recorded on individual data Proforma as yes or no. Students who do not give consent and were above or below the age of 15 year excluded from the study. Missing teeth due to trauma or non-carious reasons, and those who have anomaly or any chronic disease having medications were excluded from the study. Data was entered and analyzed using SPSS version 20. Descriptive part of the study was analyzed in the Percentage and frequency. Cross tabulation was done between the variables (socioeconomic, oral hygiene and knowledge about the disease) and presence of dental caries. The impact of demographic variables on status of carries was noted. For this purpose, parents education was an important variable. Chi square test of association was performed to see the relationship between parent's education and caries status. This regression was performed by using backward elimination method at five steps. Logistic Regression for the prediction of caries status on basis of oral hygiene habits was used. P-value $\leq 0.05$ will be taken as significant.

\section{Results}

In Logistic regression dependent variable is caries status but independent variables are significant oral hygienic factors identified in chi square test of association. This regression model was finalized at 4th step and concluded with only three significant variables. (Tables 1-6) is again about model coefficients which are significant. Cox and Snell R square also showed sufficient explained variation in dependent variable on the basis of given independent variables. (rephrase) Hosmer and Lame show highlighted that model is good for prediction. Correct classification on the basis of the finalized model is $85 \%$ which is good as cut off value is normally $70 \%$. Three significant variables are; brushing after every meal, usage of Miswak and brush after dinner are playing significant protective role from teeth decay. Final logistic model will be $\mathrm{Z}=10.503-2.602$ (Brush after every meal)-2.391 (usage of Miswak)- 2.320 (Brush after Dinner). After that equation given below can be used for probability calculation.

$$
\pi=\frac{1}{1+e^{-z}}
$$

Table 1: Different Oral Hygiene Habits \& Their Timings in Frequency\& Percentage

\begin{tabular}{|c|c|c|}
\hline Methods of teeth cleaning & Frequency & Percentage \\
\hline \multicolumn{3}{|c|}{ Brush and paste } \\
\hline Yes & 59 & 98.3 \\
\hline No & 1 & 1.7 \\
\hline \multicolumn{3}{|c|}{ Miswak } \\
\hline Yes & 6 & 10 \\
\hline No & 54 & 90 \\
\hline \multicolumn{3}{|c|}{ Powder and Manjan } \\
\hline Yes & 8 & 13.3 \\
\hline No & 52 & 86.7 \\
\hline \multicolumn{3}{|c|}{ Dental Floss } \\
\hline Yes & 8 & 13.3 \\
\hline No & 52 & 86.7 \\
\hline \multicolumn{3}{|c|}{ Water Rinsing } \\
\hline Yes & 54 & 90 \\
\hline No & 6 & 10 \\
\hline \multicolumn{3}{|c|}{ Tongue musculature } \\
\hline Yes & 12 & 20 \\
\hline No & 48 & 80 \\
\hline \multicolumn{3}{|c|}{ Combination of above } \\
\hline Yes & 39 & 65 \\
\hline No & 21 & 35 \\
\hline \multicolumn{3}{|c|}{ Timing of tooth cleaning } \\
\hline \multicolumn{3}{|c|}{ Before breakfast } \\
\hline Yes & 58 & 96.7 \\
\hline No & 2 & 3.3 \\
\hline \multicolumn{3}{|c|}{ After breakfast } \\
\hline Yes & 7 & 11.7 \\
\hline No & 53 & 88.3 \\
\hline \multicolumn{3}{|c|}{ Before lunch } \\
\hline Yes & 8 & 13.3 \\
\hline No & 52 & 86.7 \\
\hline \multicolumn{3}{|c|}{ After lunch } \\
\hline
\end{tabular}




\begin{tabular}{|c|c|c|}
\hline Yes & 4 & 6.7 \\
\hline No & 56 & 93.3 \\
\hline \multicolumn{3}{|c|}{ Before dinner } \\
\hline Yes & 9 & 15 \\
\hline No & 51 & 85 \\
\hline \multicolumn{3}{|c|}{ Before nimaz } \\
\hline Yes & 28 & 46.7 \\
\hline No & 32 & 53.3 \\
\hline
\end{tabular}

Table 2: Frequency of Cleaning Habits In Frequency \& Percentage

\begin{tabular}{|c|c|c|}
\hline Frequency of cleaning Habits & Frequency & Percentage \\
\hline \multicolumn{3}{|c|}{ Tooth Brushing } \\
\hline Once a day & 26 & 43.3 \\
\hline Twice a day & 29 & 48.3 \\
\hline Three time or more & 4 & 6.7 \\
\hline None & 1 & 1.7 \\
\hline Total & 60 & 100 \\
\hline \multicolumn{3}{|c|}{ Dental flossing } \\
\hline Once a day & 1 & 1.7 \\
\hline Twice a day & 0 & 0 \\
\hline Three time or more & 2 & 3.3 \\
\hline None & 57 & 95 \\
\hline Total & 60 & 100 \\
\hline \multicolumn{3}{|c|}{ Miswak } \\
\hline Once a day & 12 & 20 \\
\hline Twice a day & 0 & 0 \\
\hline
\end{tabular}

\begin{tabular}{|c|c|c|}
\hline Three time or more & 2 & 3.3 \\
\hline None & 46 & 76.6 \\
\hline Total & 60 & 100 \\
\hline \multicolumn{3}{|l|}{ Powder and Manjan } \\
\hline Once a day & 6 & 10 \\
\hline Twice a day & 1 & 1.7 \\
\hline Three time or more & 0 & 0 \\
\hline None & 53 & 88.3 \\
\hline Total & 60 & 100 \\
\hline \multicolumn{3}{|c|}{ Water Rinsing } \\
\hline Once a day & 4 & 6.7 \\
\hline Twice a day & 6 & 10 \\
\hline Three time or more & 35 & 58.3 \\
\hline None & 15 & 25 \\
\hline Total & 60 & 100 \\
\hline \multicolumn{3}{|c|}{ Tongue musculature } \\
\hline Once a day & 4 & 6.7 \\
\hline Twice a day & 1 & 1.7 \\
\hline Three time or more & 9 & 15 \\
\hline None & 46 & 76.7 \\
\hline Total & 60 & 100 \\
\hline
\end{tabular}

Caries free respondents were $58.3 \%$ while $41.7 \%$ had caries. 61 totals decayed teeth, 04 missing and 07 teeth were found in this study. The average DMFT in this study was 1.12 the detail is given below.

Table 3: Knowledge About the Dental Caries in Frequency \& Percentage

\begin{tabular}{|c|c|c|c|c|c|}
\hline \multirow{2}{*}{ Sr. no } & \multirow{2}{*}{ Knowledge about the dental caries } & \multicolumn{2}{|c|}{ Yes } & \multicolumn{2}{|c|}{ No } \\
\hline & & $\mathbf{F}$ & $\%$ & $\mathbf{F}$ & $\%$ \\
\hline 1 & Do you know what tooth decay is? & 48 & 80 & 12 & 20 \\
\hline 2 & Do you know, what are the common causes of teeth decay (dental caries)? & 58 & 96.7 & 2 & 3.3 \\
\hline 3 & Do you know tooth decay (dental caries) is a preventable disease? & 50 & 83.3 & 10 & 16.7 \\
\hline 4 & Do you frequently inspect your teeth in mirror? & 49 & 81.7 & 11 & 18.3 \\
\hline 5 & Do you know? What are the consequences of tooth decay (dental caries)? & 51 & 85 & 9 & 15 \\
\hline 6 & Do you know about the management \& treatment of tooth decay (dental caries)? & 53 & 88.3 & 7 & 11.7 \\
\hline 7 & $\begin{array}{l}\text { Do you go in public hospital for management \& treatment of tooth decay (dental } \\
\text { caries)? }\end{array}$ & 20 & 33.3 & 40 & 66.7 \\
\hline 8 & Do you go in private clinic for management \& treatment of tooth decay (dental caries)? & 42 & 70 & 18 & 30 \\
\hline 9 & Does dental caries affect quality of life? & 55 & 91.7 & 5 & 8.3 \\
\hline 10 & Have you ever visited dentist? & 33 & 55 & 27 & 45 \\
\hline 11 & Do you like to visit dentist if you have pain? & 43 & 1.7 & 17 & 28.3 \\
\hline
\end{tabular}




\begin{tabular}{|c|c|c|c|c|c|}
\hline 12 & Do you think, frequent visits to dentist prevent the dental caries? & 45 & 75 & 15 & 25 \\
\hline 13 & Do you know decay (dental caries) can cause loss of your tooth? & 53 & 88.3 & 7 & 11.7 \\
\hline 14 & Do you know by damaging tooth/teeth your chewing ability will affect? & 57 & 95 & 3 & 5 \\
\hline 15 & Do you know by damaging tooth/teeth quality of health will affect? & 56 & 93.3 & 4 & 6.7 \\
\hline
\end{tabular}

Table 4: Oral Hygienic Practices as risk Factors of Dental Caries

\begin{tabular}{|c|c|c|c|c|c|c|}
\hline Sr. No & Risk factor & Exposure & Cases & Control & Chi-square Value/Fisher Exact Test & $P$-value \\
\hline \multirow{2}{*}{1} & \multirow{2}{*}{ Do you brush once a day? } & Yes & 16 & 25 & 0.372 & 0.583 \\
\hline & & No & 9 & 10 & & \\
\hline \multirow{2}{*}{2} & \multirow{2}{*}{ Do you brush twice a day? } & Yes & 8 & 28 & 14.00 & $0.000^{* *}$ \\
\hline & & No & 17 & 7 & & \\
\hline \multirow{2}{*}{3} & \multirow{2}{*}{$\begin{array}{l}\text { Is it necessary to brush teeth after every } \\
\text { meal? }\end{array}$} & Yes & 11 & 33 & 18.857 & $0.000^{* *}$ \\
\hline & & No & 14 & 2 & & \\
\hline \multirow{2}{*}{4} & \multirow{2}{*}{ Is it important to take care of teeth? } & Yes & 23 & 32 & & 1.000 \\
\hline & & No & 2 & 3 & & \\
\hline \multirow{2}{*}{5} & \multirow{2}{*}{ Do you brush after breakfast? } & Yes & 4 & 6 & & 1.000 \\
\hline & & No & 21 & 29 & & \\
\hline \multirow{2}{*}{6} & \multirow{2}{*}{ Do you brush after lunch? } & Yes & 1 & 1 & & 1.000 \\
\hline & & No & 24 & 34 & & \\
\hline \multirow{2}{*}{7} & \multirow{2}{*}{ Do you brush after dinner? } & Yes & 6 & 26 & 14.816 & $0.000^{* *}$ \\
\hline & & No & 19 & 9 & & \\
\hline \multirow{2}{*}{8} & \multirow{2}{*}{ Do you brush before sleeping } & Yes & 8 & 28 & 14.000 & $0.000^{* *}$ \\
\hline & & No & 17 & 7 & & \\
\hline \multirow{2}{*}{9} & \multirow{2}{*}{$\begin{array}{l}\text { Have you ever been told the benefits of } \\
\text { tooth brushing? }\end{array}$} & Yes & 12 & 32 & 14.065 & $0.000^{* *}$ \\
\hline & & No & 13 & 3 & & \\
\hline \multirow{2}{*}{10} & \multirow{2}{*}{ Do you Miswak before nimaz? } & Yes & 8 & 30 & 18.119 & $0.000^{* *}$ \\
\hline & & No & 17 & 5 & & \\
\hline \multirow{2}{*}{11} & \multirow{2}{*}{ Do you use floss? } & Yes & 2 & 1 & & 0.565 \\
\hline & & No & 23 & 34 & & \\
\hline \multirow{2}{*}{12} & \multirow{2}{*}{ Do you use floss after breakfast? } & Yes & 0 & 1 & 1.982 & 0.664 \\
\hline & & No & 25 & 34 & & \\
\hline 13 & & Yes & 0 & 1 & & 1.000 \\
\hline 13 & Do you use floss after lunch? & No & 25 & 34 & & \\
\hline 14 & Do you use floss after dinner? & Yes & 1 & 1 & & 1.000 \\
\hline 14 & Do you use lioss arter ainner! & No & 24 & 34 & & \\
\hline 15 & Do you use floss before sleening? & Yes & 1 & 1 & & 1.000 \\
\hline 15 & Do you use lioss delore steeping? & No & 24 & 34 & & \\
\hline 16 & Do you know using fluorides tooth paste & Yes & 17 & 23 & 0.034 & 1.000 \\
\hline 16 & protect the teeth against caries? & No & 8 & 12 & & \\
\hline & Have you ever learnt techniques of tooth & Yes & 23 & 33 & & 1.000 \\
\hline 17 & brushing? & No & 2 & 2 & & \\
\hline 18 & Do you think rinsing after meal can & Yes & 20 & 30 & & 0.728 \\
\hline 18 & prevent tooth decay? & No & 5 & 5 & & \\
\hline 19 & Do you know frequently brushing can & Yes & 21 & 29 & & 1.000 \\
\hline 19 & prevent tooth decay ( dental caries) & No & 4 & 6 & & \\
\hline 20 & Do you know brushing after meal can & Yes & 23 & 31 & & 1.000 \\
\hline 20 & prevent tooth decay? & No & 2 & 4 & & \\
\hline & Do you know by cleansing teeth with & Yes & 3 & 7 & & 0.499 \\
\hline 21 & $\begin{array}{l}\text { tongue musculature can prevent tooth } \\
\text { decay? }\end{array}$ & No & 22 & 28 & & \\
\hline
\end{tabular}




\begin{tabular}{|c|c|c|c|c|c|c|}
\hline \multirow{2}{*}{22} & \multirow{2}{*}{$\begin{array}{l}\text { Do you know use of floss can prevent } \\
\text { tooth decay (dental caries)? }\end{array}$} & Yes & 0 & 2 & & 0.506 \\
\hline & & No & 25 & 33 & & \\
\hline \multirow{2}{*}{23} & \multirow{2}{*}{$\begin{array}{l}\text { What do you think Miswak is better than } \\
\text { brush? }\end{array}$} & Yes & 21 & 27 & 0.429 & 0.745 \\
\hline & & No & 4 & 8 & & \\
\hline \multirow{2}{*}{24} & \multirow{2}{*}{$\begin{array}{l}\text { What do you think brush is better than } \\
\text { Miswak? }\end{array}$} & Yes & 6 & 12 & 0.735 & 0.569 \\
\hline & & No & 19 & 23 & & \\
\hline \multirow{2}{*}{25} & \multirow{2}{*}{$\begin{array}{l}\text { Do you know use of Manjan, datin, \& } \\
\text { tooth powder can damage teeth? }\end{array}$} & Yes & 14 & 20 & 1.15 & 0.802 \\
\hline & & No & 12 & 14 & & \\
\hline \multirow{2}{*}{26} & \multirow{2}{*}{ Do you use soft brush? } & Yes & 22 & 32 & & 0.686 \\
\hline & & No & 3 & 3 & & \\
\hline \multirow{2}{*}{27} & \multirow{2}{*}{ Do you use hard brush? } & Yes & 1 & 6 & & 0.222 \\
\hline & & No & 24 & 29 & & \\
\hline \multirow{2}{*}{28} & \multirow{2}{*}{$\begin{array}{l}\text { Do you know forceful brushing can } \\
\text { damage teeth? }\end{array}$} & Yes & 11 & 17 & 0.122 & 0.796 \\
\hline & & No & 14 & 18 & & \\
\hline \multirow{2}{*}{29} & \multirow{2}{*}{$\begin{array}{l}\text { Do you think that soft brush is better for } \\
\text { teeth? }\end{array}$} & Yes & 21 & 33 & & 0.152 \\
\hline & & No & 4 & 1 & & \\
\hline \multirow{2}{*}{30} & \multirow{2}{*}{$\begin{array}{l}\text { Do you think tooth pick can damage } \\
\text { teeth? }\end{array}$} & Yes & 20 & 28 & 0.00 & 1.000 \\
\hline & & No & 5 & 7 & & \\
\hline \multirow{2}{*}{31} & \multirow{2}{*}{$\begin{array}{l}\text { Do you clean your teeth from every } \\
\text { surface? }\end{array}$} & Yes & 20 & 32 & 1.648 & 0.259 \\
\hline & & No & 5 & 3 & & \\
\hline \multirow{2}{*}{32} & \multirow{2}{*}{ Do you use mouth wash? } & Yes & 8 & 11 & & 1.000 \\
\hline & & No & 17 & 24 & & \\
\hline \multirow{2}{*}{33} & \multirow{2}{*}{ Do you use electric brush? } & Yes & 3 & 4 & & 1.000 \\
\hline & & No & 22 & 31 & & \\
\hline \multirow{2}{*}{34} & \multirow{2}{*}{ Do you use manual brush? } & Yes & 20 & 31 & & 0.470 \\
\hline & & No & 5 & 4 & & \\
\hline
\end{tabular}

Table 5: Impacts of Parental Education and financial status on caries

\begin{tabular}{|c|c|c|c|c|c|c|}
\hline Sr. No. & Risk Factor & Exposure & Cases & Control & Chi-square Value/Fisher Exact Test & $P$-value \\
\hline \multirow{3}{*}{1} & \multirow{3}{*}{ Father Education } & Low & 20 & 10 & 18.050 & 0.000 \\
\hline & & Medium & 5 & 12 & & \\
\hline & & High & 0 & 13 & & \\
\hline \multirow{3}{*}{2} & \multirow{3}{*}{ Mother Education } & Low & 17 & 8 & 14.831 & 0.000 \\
\hline & & Medium & 8 & 18 & & \\
\hline & & High & 0 & 9 & & \\
\hline \multirow{3}{*}{3} & \multirow{3}{*}{ Financial Status } & Less Than 15000 & 16 & 4 & 22.253 & 0.000 \\
\hline & & Between 15001-50000 & 9 & 20 & & \\
\hline & & More Than 50001 & 2 & 9 & & \\
\hline
\end{tabular}

Table 6: Logistic Regression for The Prediction of Caries Status on Basis of Oral Hygiene Measurements Habits Omnibus Tests of Model Coefficients.

\begin{tabular}{|c|c|c|c|}
\hline Step 4a & Chi-square & Df. & Sig. \\
\hline a) Step & -2.400 & 1 & 0.12 \\
\hline b) Block & 39.443 & 3 & .000 \\
\hline c) Model & 39.443 & 3 & .000 \\
\hline
\end{tabular}


Table 6a.

\begin{tabular}{|c|c|c|c|}
\hline Step $\mathbf{4}^{\mathrm{a}}$ & Chi-square & Df. & Sig. \\
\hline a) Step & -2.400 & 1 & 0.12 \\
\hline b) Block & 39.443 & 3 & .000 \\
\hline c) Model & 39.443 & 3 & .000 \\
\hline
\end{tabular}

Table $6 \mathrm{~b}$.

\begin{tabular}{|c|c|c|c|}
\hline Step & -2 Log likelihood & $\begin{array}{c}\text { Cox \& Snell R } \\
\text { Square }\end{array}$ & $\begin{array}{c}\text { Nagelkerke R } \\
\text { Square }\end{array}$ \\
\hline 4 & $42.060^{\mathrm{a}}$ & .482 & .649 \\
\hline
\end{tabular}

Note: Model Summary

Table 6c.

\begin{tabular}{|c|c|c|c|}
\hline Step & Chi-square & Df & Sig. \\
\hline 4 & 1.066 & 4 & .900 \\
\hline
\end{tabular}

Note: Hosmer and Lemeshow Test

Table 6d.

\begin{tabular}{|c|c|c|c|}
\hline \multirow{2}{*}{ Observed } & \multicolumn{2}{|c|}{ Predicted } \\
\cline { 2 - 4 } & \multicolumn{2}{|c|}{ Does subject has caries? } & \multirow{2}{*}{ Percentage Correct } \\
\cline { 2 - 4 } & \multicolumn{2}{|c|}{ Yes } & No \\
\hline Step 4 Does subject has caries? & & 7 & 72 \\
\hline Yes & 18 & 33 & 94.3 \\
\hline No & 2 & & 85.0 \\
\hline Overall Percentage & & 73 \\
\hline
\end{tabular}

Note: Classification Table

Table 6e.

\begin{tabular}{|c|c|c|c|c|c|c|c|c|c|}
\hline \multirow{2}{*}{\multicolumn{2}{|c|}{ Step $4^{\mathrm{a}}$}} & \multirow{2}{*}{ B } & \multirow{2}{*}{ S.E. } & \multirow{2}{*}{ Wald } & \multirow{2}{*}{ Df. } & \multirow{2}{*}{ Sig. } & \multirow{2}{*}{$\operatorname{Exp}(B)$} & \multicolumn{2}{|c|}{ 95.0\% C.I.for EXP(B) } \\
\hline & & & & & & & & upper & Lower \\
\hline a) & Brush after every meal & -2.602 & .888 & 8.584 & 1 & .003 & .074 & .013 & .423 \\
\hline b) & Usage of Miswak & -2.391 & .906 & 6.965 & 1 & .008 & .092 & .015 & .540 \\
\hline c) & Brush after Dinner & -2.320 & 1.005 & 5.329 & 1 & .021 & .098 & .014 & .704 \\
\hline & d) Constant & 10.503 & 2.583 & 16.537 & 1 & .000 & $3.643 \mathrm{E} 4$ & & \\
\hline
\end{tabular}

Note: Variables in the Equation

Table $6 f$.

\begin{tabular}{|c|c|c|c|}
\hline \multicolumn{1}{|c|}{$\begin{array}{c}\text { Step 4 } \\
\text { Variables }\end{array}$} & Score & Df \\
\hline a) Twice day brushing & 2.430 & 1 & .119 \\
\hline b) Brushing before sleeping & .002 & 1 & .966 \\
\hline c) Benefits of brushing & 1.067 & 1 & .302 \\
\hline d) $\quad$ Overall Statistics & 3.210 & 3 & .360 \\
\hline
\end{tabular}

Note: Variables not in the Equation

\section{Discussion}

Objective of the study was to see the impact of oral hygienic practices on tooth decay. Different practices have always strong influence on diseases patterns, their intensities and their further complications. This study has explored that there are few practices which have significant relationship with the caries. This study has shown that brushing twice a day and after every meal, brushing after dinner and before sleeping, using Miswak and having awareness about teeth protection or brushing were the significant information's or practices for avoiding teeth decay. Many of questions are directly or indirectly linked with brushing habits so these habits were played protective role in this study. Insignificant practices might be due to less prevalence of these practices in teenagers; like Manjan and Datin etc., are some other researches have also supported this argument that oral hygienic practices play significant role in avoiding dental caries [13-15]. Total fifty thee percent $(58.3 \%)$ respondents were caries free in this study while caries positive were $41.7 \%$. Average DMFT score examined was 1.2. 
Similar result was found in a study conducted by Kiran Kumar in 2013 and the mean DMFS was $1.2 \pm 0.79 .16$ Results of this study did not match with the studies like; the mean DMF-T index was 4.6 by Amaral et al. [17]; average DMFT was 3.27 by Mustahsen et al. [18]; average DMFT was 2.93 by Carlos et al. [19]; mean DMFT was 3.7 by Abdullah [20]; the mean DMFT was 0.14 by Adekoya-Sofowora et al. [21]; The mean DMFT was 0.53 by Sohi et al. [22]; mean DMFT was 0.97 by Hou et al. [23]; DMFT score was 1.4 by Leghari et al. [24]; the mean DMFT was 3.70 by Ali et al. [25]; and mean DMFT score was $2.22 \pm 2.19$ by Ali et al. [26]. This study has explored that father and mother education has strong positive impact on caries. As parents have higher level of education there will be higher chances to avoid from caries or other teeth problems and vice versa. It is simple that if parents have higher level of education then they will be better aware from diseases and their management. So, their education play significantly protective role for caries status. Similarly, economic background or financial position matters in this study. As higher the financial position lower will be the chances of getting this disease of caries. These are few researches which have supported this argument that demographic has strong impact on caries status is the relevant studies in this case $[15,27]$.

\section{Conclusion}

Findings of this study have shown that brushing twice a day and after every meal, brushing after dinner and before sleeping, using Miswak and having awareness about teeth protection or brushing were the significant data or practices for avoiding teeth decay. Other practices have insignificant or less significant role in this study. This study has explored that father's and mother's education level has strong positive impact on caries. As parents have higher level of education there will be fewer chances to have caries or other teeth problems and vice versa. So, their education plays a significantly protective role for caries status. As higher the financial position, lower are the incidence of dental caries.

\section{Some Recommendations to Reduce Burden of Dental Caries in Local Settings}

a. Changes in lifestyle ought to adopt like reduce the intake of food containing free sugars to four times a day and thereby limit the amount of free sugars consumed. Individuals need to encourage using fluoride toothpaste twice a day after meal. Use of Dental floss, rinsing with water after meal, and cleanings of teeth with tongue after every meal can also reduce incidence of caries. Regular visit to dentist is recommended.

b. Pakistan is currently undergoing nutrition transition do not have adequate exposure to fluoride. There is need to promote adequate fluoride exposure via appropriate vehicles, for example affordable toothpaste, water, salt and milk. Guidelines for health promotion and disease prevention should be incorporated in the syllabus of pre-school and school level.

c. Availability of health care center and human resource for management of the dental caries ought to ensure in every union council. Referral system of affected individuals should be initiated from different schools, institute and organization to health care center for secondary prevention of dental caries by restoration of the affected teeth.

d. Health education campaigns and health promotion websites need to encourage which are particularly famous in youth population. Mass media campaigns have to start for oral health promotion in order to reduce dental caries incidence. Oral hygiene campaign needs to start in our community to reduce dental caries prevalence in the; schools' colleges, universities and madrasas. Services of various health promotion programs can be utilized to reduce dental caries incidence and prevalence.

\section{Reference}

1. Petersen PE, Ogawa H (2016) Prevention of dental caries through the use of fluoride-the WHO approach. Community Dent Health 33(2): 6668.

2. World Health Organization (2016): Global Oral Health Data Bank. Geneva: WHO.

3. Haque SE, Rahman M, Itsuko K, Mutahara M, Kayako S, et al. (2016) Effect of a school-based oral health education in preventing untreated dental caries and increasing knowledge, attitude, and practices among adolescents in Bangladesh. BMC oral health 16(1): 44.

4. Kwan S, Petersen PE (2010) Oral health: equity and social determinants. Equity, social determinants and public health programmes 39(6): 159176.

5. Marinho VC, Chong LY, Worthington HV, Walsh T (2016) Fluoride mouthrinses for preventing dental caries in children and adolescents. Cochrane Database of Systematic Reviews.

6. Van der Walt M, Van Wyk PJ, Bester JK, Becker PJ (2018) The effectiveness of a tooth brushing programme for children in the Ehlanzeni district of Mpumalanga. South African Dental Journal 73(10): 604-611.

7. Vanobbergen J, Martens L, Lesaffre E, Bogaerts K, Declerck D, et al. (2001) Assessing risk indicators for dental caries in the primary dentition. Community dentistry and oral epidemiology 29(6): 424-434.

8. Varenne B, Petersen PE, Ouattara S (2006) Oral health behaviour of children and adults in urban and rural areas of Burkina Faso, Africa. International dental journal 56(2): 61-70.

9. Ganavadiya R, Chandrashekar BR, Goel P, Hongal SG, Jain M, et al. (2014) Mobile and portable dental services catering to the basic oral health needs of the underserved population in developing countries: A proposed model. Annals of medical and health sciences research 4(3): 293-304.

10. Barnes VM, Richter R, DeVizio W (2010) Comparison of the short-term antiplaque/antibacterial efficacy of two commercial dentifrices. J Clin Dent 21(4): 101-104.

11. Garcia RI (2008) Mouthrinses and dentifrices are effective antigingivitis and antiplaque agents. Journal of Evidence Based Dental Practice 8(1): 13-14

12. Halawany HS, Abraham NB, Jacob V, Al Maflehi N (2015) The perceived concepts of oral health attitudes and behaviors of dental students from four Asian countries. The Saudi Journal for Dental Research 6(2): 79-85.

13. Caries D Selwitz RH, Ismail AI, Pitts NB (2007) Lancet 369: 51-59.

14. Löe $H$ (2000) Oral hygiene in the prevention of caries and periodontal disease. International dental journal 50(3): 129-139.

15. Shah N, Sundaram KR (2004) Impact of socio-demographic variables, oral hygiene practices, oral habits and diet on dental caries experience of Indian elderly: a community-based study. Gerodontology 21(1): 43-50. 
16. Kumar K (2013) Patterns and distribution of dental caries and dental fluorosis in areas with varying degrees of fluoride ion concentration in drinking water. Journal of Oral Hygiene \& Health 3: 1-7.

17. Amaral MA, Nakama L, Conrado CA, Matsuo T (2005) Dental caries in young male adults: prevalence, severity and associated factors. Brazilian oral research 19(4): 249-255.

18. Ur Rehman MM, Mahmood N, Ur Rehman B (2008) The relationship of caries with oral hygiene status and extra-oral risk factors. J Ayub Med Coll Abbottabad 20(1): 103-108.

19. Tambelini CA, Ramos DM, Poli Frederico RC, Tomasetti CS, Barata TD, et al. (2010) Dental caries in adolescents and its association with excess weight and sociodemographic factors in Londrina, Paraná, Brazil. Revista Odonto Ciência 25(3): 245-249.

20. Abdullah HA (2009) Prevalence of dental caries and associated teeth brushing behavior among Iraqi adolescents in Al-Door district. Medical Journal of Tikrit 2(152): 102-109.

21. Adekoya Sofowora CA, Nasir WO, Oginni AO, Taiwo M (2006) Dental caries in 12-year-old suburban Nigerian school children. African Health Sciences 6(3): 145-150.

ISSN: 2574-1241

DOI: 10.26717/BJSTR.2019.20.003469

Rasul F. Biomed J Sci \& Tech Res

(C) (P) This work is licensed under Creative

Submission Link: https://biomedres.us/submit-manuscript.php
22. Sohi RK, Gambhir RS, Veeresha KL, Randhawa AK, Singh G, et al. (2012) Assessment of prevalence of dental caries among 5 and 12-year-old schoolchildren in Chandigarh (UT), India. Archives of Oral Research $8(1)$.

23. Hou R, Mi Y, Xu Q, Wu F, Ma Y, et al. (2014) Oral health survey and oral health questionnaire for high school students in Tibet, China. Head \& face medicine $10(1): 17$.

24. Leghari MA, Tanwir F, Ali H (2014) Association of dental caries and parents knowledge of oral health, A cross-sectional survey of Private Schools. J PAk Dent Assoc 23(1): 19-24.

25. Ali s, Dastgir Bhatti Mu, Syed A, Chaudhry Au, Iqbal Z, et al. (2012) Prevalence of dental caries among 5-14 years old poor locality school children of lahore. Pakistan Oral \& Dental Journal 32(2).

26. Ali A, Asghar S, Somoro S (2013) Caries prevalence among school children age 6-14 years in Gadap town Karachi in relation to the awareness of their parents toward oral health. Pak Oral Dent J 33(2): 354-358.

27. Gibson S, Williams S (1999) Dental caries in pre-school children: associations with social class, toothbrushing habit and consumption of sugars and sugar-containing foods. Caries research 33(2): 101-113.

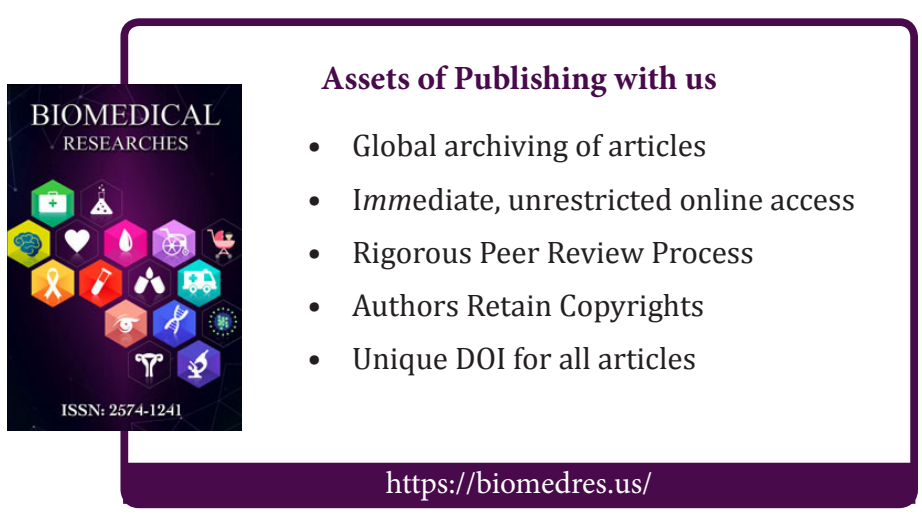

